\title{
Letter to the Editor for the Manuscript the Complex Interplay of Physical Fitness, Protein Intake and Vitamin D Supplementation After Bariatric Surgery
}

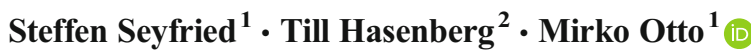

Published online: 16 September 2017

(C) Springer Science+Business Media, LLC 2017

Dear editor,

We agree with the authors that postoperative body composition and maintenance of muscle mass has an influence on the results of bariatric surgery. Preoperative handgrip strength and phase angle (which is part of the bioelectrical impedance analysis, reflecting quality of the lean body mass) are strongly correlated with postoperative body composition changes and even weight lossthis means that physical functionality plays an important role $[1,2]$.

As the authors mentioned, there are multiple factors responsible for skeletal muscle atrophy and loss of bone mass. However, if the application of proteins and vitamin D will change something on the long-term course is completely unclear. Metcalf et al. showed that a postoperative exercise program can positively influence body composition, that means there are possibilities of influencing [3]. Long-term data does not exist.

We agree that the overall supplementation in bariatric patients is not sufficient. Therefore, a structured postoperative follow-up, including frequent clinical examination and investigation of the vitamin serum levels is

Mirko Otto

mirko.otto@umm.de

1 Department of Surgery, University Medical Center Mannheim, University of Heidelberg, Mannheim, Germany

2 Department of Surgery, Alfried Krupp Krankhenhaus, Essen, Germany

very important. Several published studies confirmed, that many patients are not adherent to a follow-up program [4]. As described, a structured follow-up program after bariatric surgery is crucial to improve and sustain weight loss [5]. Whether the body composition is influenced positively by such a program is unclear.

\section{Compliance with Ethical Standards}

Conflict of Interest The authors declare that they have no conflicts of interest.

Ethical Approval Does not apply.

Statement of Informed Consent Does not apply.

\section{References}

1. Vassilev G, Hasenberg T, Krammer J, et al. The phase angle of the bioelectrical impedance analysis as predictor of post-bariatric weight loss outcome. Obes Surg. 2017;27(3):665-9.

2. Otto M, Kautt S, Kremer M, et al. Handgrip strength as a predictor for post bariatric body composition. Obes Surg. 2014;24(12):20828.

3. Metcalf B, Rabkin RA, Rabkin JM, et al. Weight loss composition: the effects of exercise following obesity surgery as measured by bioelectrical impedance analysis. Obes Surg. 2005;15(2):183-6.

4. Vidal P, Ramon JM, Goday A, et al. Lack of adherence to follow-up visits after bariatric surgery: reasons and outcome. Obes Surg. 2014;24:179-83.

5. Elrefai M, Hasenberg T, Vassilev G, et al. Adherence to a follow up program is improving weight loss. Bariatric Surgical Practice and Patient Care. 2017. https://doi.org/10.1089/bari.2017.0019. 